\title{
Cognitive Computing and wireless Communications on the Edge for Healthcare Service Robots
}

\author{
Shaohua Wan ${ }^{\mathrm{a}, \mathrm{b}, *}$, Zonghua $\mathrm{Gu}^{\mathrm{c}, \mathrm{d}}$, Qiang $\mathrm{Ni}^{\mathrm{e}}$ \\ ${ }^{a}$ School of Information and Safety Engineering, Zhongnan University of Economics and \\ Law, Wuhan 430073 China. \\ ${ }^{b}$ State Key Laboratory for Novel Software TechnologyNanjing University, Nanjing 210023, \\ China. \\ ${ }^{c}$ Department of Applied Physics and Electronics, Umeå universitet, 90187 Umeå, Sweden. \\ ${ }^{d}$ College of Computer Science, Zhejiang University, Hangzhou 310027, China. \\ ${ }^{e}$ School of Computing and Communications, Lancaster University, Lancaster, LA1 $4 W A$, \\ $U K$.
}

\begin{abstract}
In recent years, we have witnessed dramatic developments of mobile healthcare robots, which enjoy many advantages over their human counterparts. Previous communication networks for healthcare robots always suffer from high response latency and/or time-consuming computing demands. Robust and high-speed communications and swift processing are critical, sometimes vital in particular in the case of healthcare robots, to the healthcare receivers. As a promising solution, offloading delay-sensitive and communicating-intensive tasks to the robot is expected to improve the services and benefit users. In this paper, we review several state-of-the-art technologies, such as the human-robot interface, environment and user status perceiving, navigation, robust communication and artificial intelligence, of a mobile healthcare robot and discuss in details the customized demands over offloading the computation and communication tasks. According to the intrinsic demands of tasks over the network usage, we categorize abilities of a typical healthcare robot into alternative classes: the edge functionalities and the core functionalities. Many latency-sensitive tasks, such as user interaction, or time-consuming tasks including health receiver status recognition and
\end{abstract}

\footnotetext{
* Corresponding author

Email addresses: shaohua.wan@ieee.org (Shaohua Wan), zonghua.gu@umu.se (Zonghua $\mathrm{Gu}), \mathrm{q} \cdot \mathrm{ni}$ @lancaster.ac.uk (Qiang Ni)
}

Preprint submitted to Journal of ${ }^{A} T_{E} X$ Templates

October 8, 2019 
autonomous moving, can be processed by the robot without frequent communications with data centers. On the other hand, several fundamental abilities, such as radio resource management, mobility management, service provisioning management, need to update the main body with the cutting-edge artificial intelligence. Robustness and safety, in this case, are the primary goals in wireless communications that AI may provide ground-breaking solutions. Based on this partition, this article refers to several state-of-the-art technologies of a mobile healthcare robot and reviews some challenges to be met for its wireless communications.

Keywords: healthcare robot, wireless communication, edge computing, artificial intelligence.

\section{Introduction}

Edge computing is expected to be a key enabler of processes where a rapid response to sensor input is necessary, such as wireless health monitoring, virtual reality, and robotics. Providing such healthcare gets expensive on a daily basis,

5 especially for the elder population around the world. This is important in particular when dealing with chronic and psychological diseases. Nursing and carereceiving often require long-term intensive human labor. Robotics, as a promoting solution, has open a way to explore a constantly-accompanying and automatic caregivers to help provide "a mobile healthcare robot".

A mobile healthcare robot enjoys enormous superiorities over human-labor in healthcare, including but not limited to the following. Under the support of artificial intelligence, a robot can learn massive intelligence and experience from human medical experts, or sometimes even outperforms their human counterparts. Such intelligent robots can provide more efficient diagnosis and treatment than a human caregiver. Subsequently, a healthcare robot either autonomously moves or is incorporated into a mobile digital device. Mobility of the robot means superior adherence anywhere at any time. For example, a psychological therapy robot can be a table-top device connected with a smart phone [1]. 
Afterwards, a robot is equipped with various sensors and enabled to capture excessively more details of the care-receiver and the environment than a human care-giver. The captured information is vital for improving the health condition of a care-receiver [2]. Finally, a robot is more adept at repetitive work and less error-prone than humans. For instance, a robot can remind a care-receiver of the medication schedule at any given time [3].

Efficient communications between robots and data centers are essential for improving customer services. However, the risk of potential high response latency at the data center end is critical for healthcare robot, especially in the case of emergency aid. In addition, many time-consuming tasks, such as human understanding. Such tasks can be completed by the robot and only summarized messages. Such messages as the category of behaviors, are communicated with the centers with very limited communication burden. A careful design of the framework in balancing edge computing and centralized computing is important for researchers from both robotics and communication communities [4] [5] [7].

The rapid development of Internet of Things (IoT) [8 9 [10 is getting a wide acceptance and a growing adoption in many aspects of our daily life. By applying IoT technologies to healthcare, it is expected to witness dramatic improvement of healthcare and thus increase the service quality to humans [12] 13] [17. To release the heavy communication burden of healthcare robots, in particular from their various equipment. It is preferred to pre-process the huge amount 40 of data by the robots, or the edge computing [11 18, 19] 20] 14, 16. In this paper, we aim to introduce applications of edge computing scenarios of mobile healthcare robots and give details on edge/centralized computing analysis in a task-driven fashion. The main contribution of this paper are as follows:

- Investigate on the application and key technologies of mobile healthcare 45 robots.

- Discuss and analyze several peripheral and core functionalities that the robot development will require.

- Embracing edge computing and healthcare robots in image understanding, 
sensor and path planning technologies will speedup the progress toward

practice.

The rest of this paper is organized as follows. Section 2 summarizes typical needs and functionalities of the robot. Section 3 points out the task-driven demands of healthcare robots in edge computing. Section 4 analyzes the centralized case. Section 5 gives the conclusion and future work.

\section{Typical Applications of A Mobile Healthcare Robots}

The result of edge computing can be rapid machine-to-machine communication or machine-to-human interaction. This paradigm takes localized processing farther away from the network right down to the sensor by pushing the computing processes even closer to the data sources. The sensor can act as a dispatcher that can send information to another edge device or to the cloud if need be. This allows each edge device to do its part in processing information instead of sending all its data to a centralized server. Edge computing help improve patient care as well as increase efficiency from a business perspective. By spreading out the network, organizations can enhance productivity by concentrating resources

65 on certain tasks and making health IT systems more efficient by decentralizing IT infrastructure. Fig. 1 illustrates representative application scenarios and functionalities of a mobile healthcare robot. We have listed several applications that a mobile healthcare robot may fit in.

\subsection{Elder and Chronic Patient Nursing}

70

Seventy percent of U.S. citizens take at least one prescription medication and over fifty percent take at least two, according to FDA and CDC. Among these patients, forty percent arises from elders. Failing to maintain medication adherence is a dramatic barrier to pursue health for patients, in particular those of elder people or chronic patients. A typical task consists of detection, commu75 nication with cloud, processing and returning message. A healthcare robot in this case is expected to be able to detect any abnormal actions, such as falling 


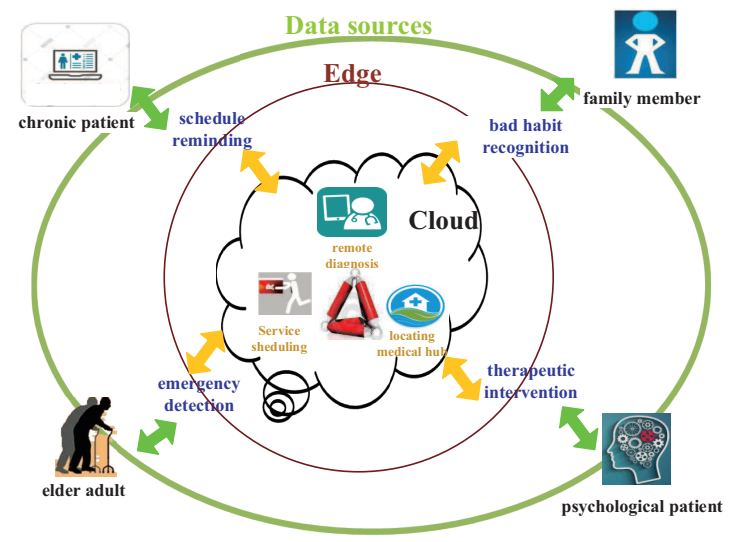

Figure 1: Needs on mobile healthcare robots

down, faint or asking for help. The robot then processes the raw detections and uploads compressed information to the cloud by reliable wireless connections. Messages are sent back to the robot to act properly. Frequently missing of medication doses, consequently and unfortunately, will likely lead to diseases aggravation.

\subsection{Unhealthy Habit Recognition}

Healthy lifestyle plays an important role in health maintenance. Harmful habits may lead a person towards unhealthy conditions even if he/she is temporarily healthy. However, a habit is an unconscious behavior. One usually fails to realize that a negative habit is doing harm. Professional suggestions, in this case, are essential for early-preventing.

\subsection{Mental Healthcare}

Although psychological issues like depression are increasingly prevalent, many people still face high barriers to access mental healthcare facilities. Some suffers do not realize the necessity of mental healthcare for fear of the social stigma associated with receiving psychotherapy. Other suffers desire healthcare but are impeded by high financial costs of mental health services. 


\section{Edge-Computing-Friendly Functionalities}

a robot are edge-computing-friendly in nature. In this section, we list and analyze this kind of tasks and discuss the corresponding edge computing techniques. In general, many user-orientated applications, such as user-friendly interfaces, intelligent perceptions, automatic navigations and innovations, as shown in Fig. center.

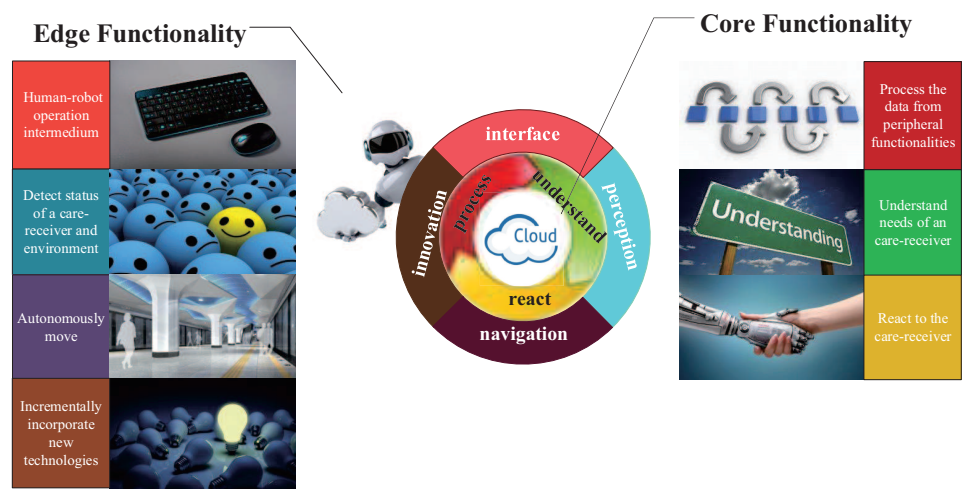

Figure 2: Key technologies of mobile healthcare robots in edge/centralized computing.

\subsection{Interactivities}

Typical interactivities between a robot and its users are commonly accomplished by the robot although several intelligent interfaces are more data-centerorientated. Traditional reaction of a robot to a human behavior is often defined off line and fixed in operation. Very limited communications, always a predefined message in describing user activities is sent to the center for services' improvement. However, as the rapid growth of customized services with support from the cutting-edge AI techniques, robot-human interactivity demands more communications than before 21. For example, Google Siri, a popular virtual assistant, employs the state-of-the-art AI and machine learning technologies to recognize speech and answer questions and prefer to link to the data center for 
an accurate reaction. It is a common choice in using the combination of an on-line and off-line interactive algorithms [29], in which in common scenes, the well-trained off-line model is adapted for the purpose of efficiency and on-line is selected in some special applications. Human-robot interactivities are also the main source of data stream in an edge computing environment, where the state-of-the-art resource allocation [23] or computation offloading strategy [24] can be applied.

\subsection{Perception}

Perception is capable of capturing passive inputs and enhances the ability of data collection. This edge function should preliminarily understand the perceived data, which is the basis of further analysis on the cloud.

Mobile-phone-based method: A virtual agent installed in a smart phone can

directly collect incoming and outgoing text messages of all online-chatting APPs [1. Thus, the virtual agent can simply perceive the user's activities through text messages. Obviously, this method is limited to text information and inevitably omits a lot of user behaviors, which may be important, sometimes even fatal, to healthcare-receivers. The virtual agent extends its perception capability by capturing the user's facial expressions using the front camera of a smart phone.

Facial recognition: Under the support of facial recognition technology, a tabletop healthcare robot can monitor each family member and his or her medications [3]. The robot reminds patients in pursuing their medication schedule, recognizes a patient's health crisis and contacts a healthcare provider if necessary. Moreover, such robot is enabled to connect to wireless networks and serves as interchanging communication medium between patients and healthcare providers. State-of-the-art facial recognition technologies can further improve recognition accuracy [27].

Special sensors: Human activity recognition is an essence to enable a robot to identify the behavior of a specific care-receiver [28]. Rather than facial expressions, an activity recognition can perceive behaviors of a care-receiver, who may be an elder adult, a children or a chronic patient. By activity recognition, 
a robot tacks the care-receiver's action and recognize human behaviors such as anomalous activities and unhealthy habits. To ensure a robust and accurate recognition, sensors like accelerometers and gyroscopes are important in autonomously detecting human behaviors under certain scenarios.

Although the equipment of special sensors may improve human activity recognition performance, those sensors are still not user-friendly and, as a result, not welcome in industry [22]. Special sensors are typically not pressurefree to the wearer, which means low comfort level or restriction of the carereceiver mobility. In addition, deployment and maintenance of such sensors commonly induce heavy financial burden. Consequently, a more feasible approach is camera surveillance and image-classification-based human behavior recognition. Nevertheless, recognizing human activities solely from images is an extremely challenging task. Many challenges, such as background disarray, diversity of viewpoint, resemblance of distinct human behavior, may dramatically depress classification performance. Thanks to the rapid development of cutting edge machine learning schemes, promising solutions may arise from several state-of-the-art deep learning algorithms, including Convolutional Neural Network (CNN) 30], Generative Adversarial Network (GAN) 31].

CNN and GAN models enable robots make informed decisions based on the tasks they're presented with. CNN-based framework is able of navigate an endovascular surgery robot based on surgeons' skill learning. CNN-based method shows its capability of adapting to different situations and achieves similar success rate and average operating time. Robotic operation performs similar operating trajectory and maintains similar level of operating force with manual operation. The CNN-based method can be easily extended to many other surgical robots. A semi-supervised learning approach with generative adversarial networks GANs that enables a robot to learn from unlabeled tactile 170 sensory data from interactions with everyday objects. By leveraging unlabeled sensor data that are more abundant in unstructured environments, we mitigate the need for massive labeled training sets. [52, 55]

The application of deep learning in robotics has also greatly improved the ac- 
curacy of the robot's work. The robot's understanding of complex environments is the first step in intelligence. Vision-based scene recognition and understanding is important to the robot's understanding of the surrounding environment and improving its intelligence level. Obtaining real-time data in the current environment is of great importance for the robot to construct the current working environment map. It is also necessary to consider the situation of the robot in the room. It is necessary to realize the correlation between the indoor threedimensional map and the semantic information, not only the aforementioned ring needs to be considered. The map of the environment also needs to be classified to identify the scene in the scene.

Feature extraction is a key step in scene recognition. In this step, we do not use the traditional method of applying local features through human intervention, but apply the convolutional neural network model in deep learning to the scene recognition of the robot so that it can automatically capture the hidden in the original image. Number of feature information according to. In the process of object recognition and large-scale natural scene image processing, the convolutional neural network and superpixels can be combined with the depth Boltzmann machine respectively, wherein the large-scale scene image is preprocessed by the convolutional neural network to obtain a volume. After the product feature, the result is used as the depth visual layer input of the Boltzmann machine, feature extraction, and then use the softmax classifier implements the classification of the scene. In the indoor scene, it is necessary to realize the correlation between the three-dimensional map and the semantic information in the room, and use the decentralized modular technology to enable the robot to simultaneously perform scene object recognition and map reconstruction, thereby realizing its indoor recognition function.

\subsection{Navigation}

Navigation is a user-specific function and directly determines the behavior of the robot. Consequently, navigation is an edge functionality. However, this functionality may need support from the cloud. 
Some mobile healthcare robots need to autonomously move, especially when

novel path planning methods.

\subsection{Innovation}

Similar to navigation, innovation is also tightly tied to user requirements. This functionality furnishes an upgrading interface for the whole system. In the that effectively facilitates a remotely teleoperated mobile health robot at home [37, 48, 49, 50, 51, 53, 54. This kind of work interprets the robot developing task from the perspective of software engineering. Systematic technology renovation 
like teleoperated healthcare robot should consistently fit into the requirements

The development of integrated information architecture, which may link with health professionals or technical personnel, is necessary for healthcare robots. The main challenge is prioritizing various possible functionalities of a robot and handling the complexity of home physical environments. The main constraints include limits on the structural, perceptual and processing technologies of the robot. A teleoperated robot is a realistic choice that leverages currently mature technologies and depends on human operations to overpass existing limitations.

A mobile healthcare robot is a data-centric system. A usable and extensible system supports all information flows and their integration fitting into a consistently integrated, unitary and secure information system. In this manner, this architecture enables all stakeholders to felicitously access the system at any proper moment.

\section{Data-Center-Orientated Communications}

Core functionalities rely on the support of artificial intelligence techniques, ${ }^{50}$ including machine learning, semantic model, sentiment analysis and so on. These techniques put forwards high demands on hardware platform as well as artificial intelligence abilities. To meet the extreme requirements for user experience, efficiency, performance in wireless robot networking environments, novel designs, configurations and optimizations for wireless communications and networking are in great need to satisfying the service requirements. As a result, the core functionalities run on the cloud and provide support to the edge functionalities. Fig. 3 summarizes the core functionalities and representative supporting technologies.

\subsection{Uncertainty handling}

Uncertainty handling is a critical issue especially when we refer to healthcare tasks, in which an unexpected operation may cause disastrous consequences. 

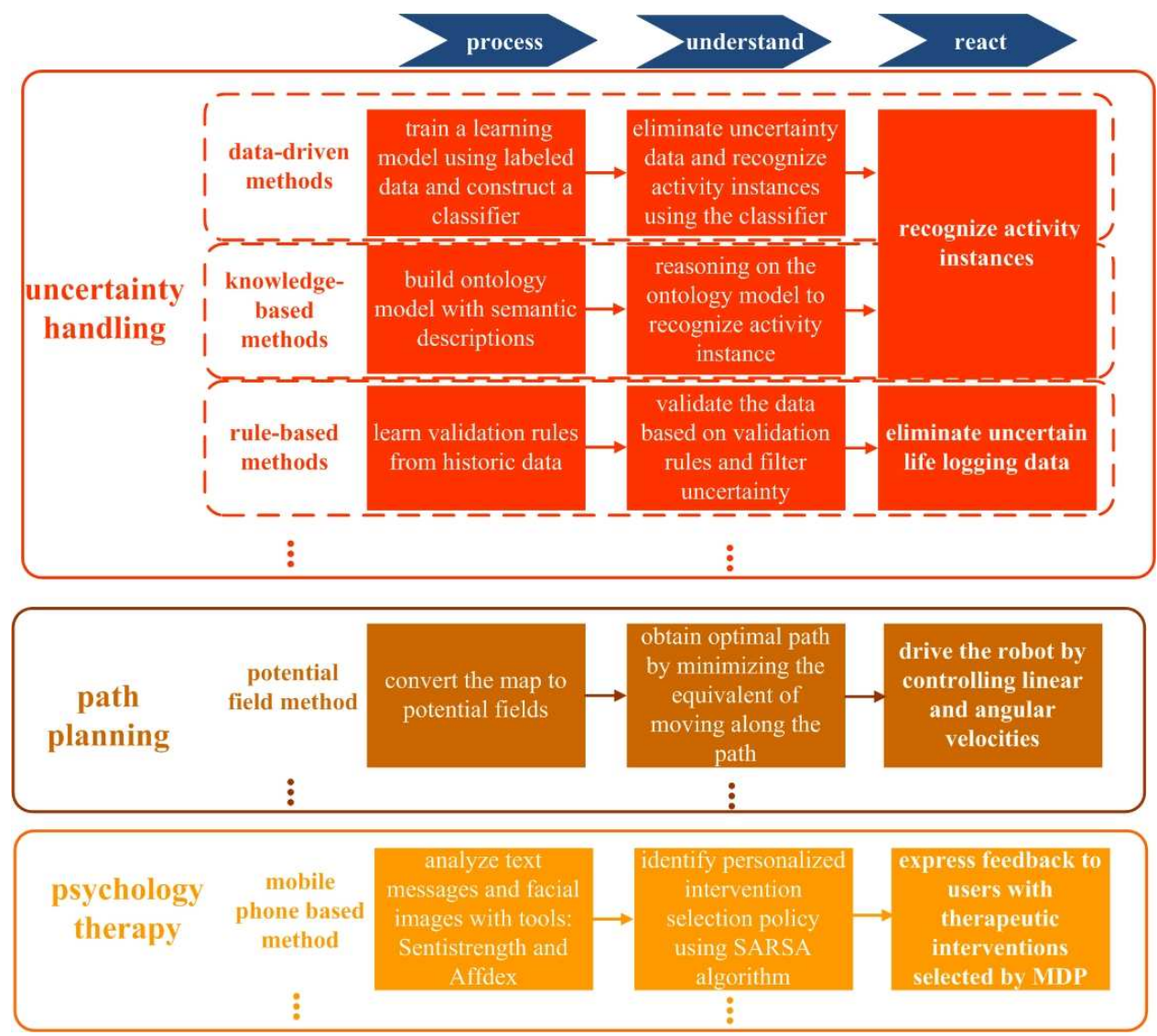

Figure 3: Core functionalities and representative supporting technologies 
As an example, Fig. 4 demonstrates sensor uncertainty in a mobile healthcare robot system. Edge computing can solve the inefficiency of moving all data to a centralized point by creating a network of smaller datacenters with dedicated purposes and features that are tailored to meet specific demands. Digital projects that create or require data can be processed much faster when the computing power is close to the device or person generating it. By spreading out the network, organizations can enhance productivity by concentrating resources on certain tasks and making health IT systems more efficient by decentralizing IT infrastructure.

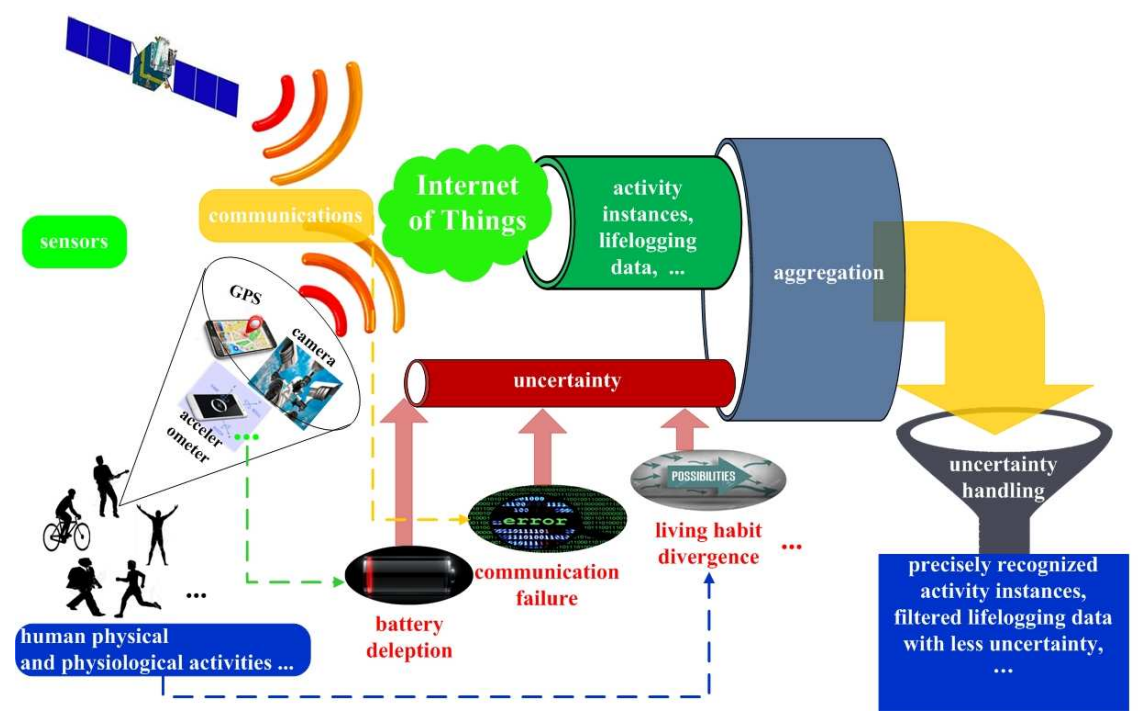

Figure 4: Sensor uncertainty in a mobile healthcare robot system

Inevitability of sensor uncertainty: Sensors suggest a promising solution to convey human's physical, physiological or even psychological activities to a robot. Internet of Things (IoT) integrates heterogeneous wearable or mobile sensors and creates a huge amount of opportunities to recognize human activities and collect human life logging data. With the support of IoT, physical activities can be remotely logged. Consequently, care-receivers are able to obtain more opportunities to enjoy a personalized healthcare.

Nevertheless, leveraging IoT in healthcare systems is challenging due to the 
fact that various sensors (wearable devices) of IoT are generating massive highdimensional heterogeneous data all the time. Effectively validating such data becomes an essential task. Owing to advances in accelerometer technologies and GPS, physical activities are generally well-observed. Life-logging physical activity data (LPD) exhibits remarkable uncertainty due to various reasons such as diversity and alterations of personal living habits, sensor errors (battery depletion, inaccurate outputs, etc.) and communication malfunction. Consequently, a mobile healthcare robot is required to make right decisions based on uncertain inputs, i.e., incomplete and inaccurate sensor data. The next generation of communication networks, such as the global centralized Software Defined Network (SDN) 41] 42, provide robust link for IoT connections. Other cutting-edge technologies, such as cognitive radio sensor networks [35], may also improve the robustness and efficiency of a public network.

Knowledge-based and Data-driven Solutions: In order to recognize human activities and handle sensor uncertainty, popular solutions always adopt datadriven or knowledge-based methods. The primary superiority of data-driven methods is their capability to deal with uncertainty. Knowledge-based methods utilize prior knowledge to construct semantic activity models and perform inference processes on input sensor data. Such methods enjoy superior interoperability and wide adaption to diversified application scenarios, which are vital for a context-aware system. Additionally, knowledge-based methods leverage formal data structures to denote sensor data and contexts under the support of semantic descriptions, which make sensor data and contexts comprehensible to both developers and robots. Ontology-based activity recognition is a typical knowledge-based method and possesses advantages of expressiveness and comprehensive reasoning mechanisms [39, 46, 47]. A barrier to its broad application is the imperfect observations which may depress the activity recognition performance.

Data-driven and knowledge-based methods have shown their success in many applications. Data-driven methods adopt supervised machine learning algorithms to categorize sensor data into groups, each of which represents one kind 
of human activity. For instance, Hidden Markov Models (HMM) and Support Vector Machine (SVM) are two widely used classifiers. Despite the success of data-driven methods, such methods fail to work efficiently with limited size of training data because they require large volume of training data to guarantee the classification accuracy. Moreover, it is difficult to acquire adequate training data because users may implement activities in various ways. In addition, gathering and manually labeling large volume of sensor data are known for their tremendous time-consumption. Furthermore, data-driven methods are difficult to process high-dimensional data.

In view of disadvantages of data-driven and knowledge-based methods, a combination of both methods enjoys broad prospects. Some recent works design hybrid models to recognize human activities. However, existing hybrid models lack specialized solutions to uncertainty handling. A hybrid model named AGACY Monitoring can cope with the inherent uncertainty of sensor data. This model handles long-enduring activities and their uncertainty values 325 by adopting a new feature extraction method. Along with this model, a novel algorithm called AGACY infers activities by probing the collected uncertainty values [38. Currently, the primary drawback of this method is that it lacks the ability to reuse existing upper ontology like DOLCE ontology.

A validation-rule based method can eliminate irregular uncertainty as well 330 as relieve the negative influence of regular uncertainty [2]. This kind of methods still faces some challenges despite its success in experiments. First, extensibility should be enhanced to flexibly incorporate new validation rules. Second, a formal rule of human-in-loop validation needs to be investigated so that the method can more efficiently leverage user feedbacks to update validation rules. 335 Third, the flexibility of the method needs to be validated by more users.

\subsection{Social-aware Path Planning}

A mobile healthcare robot may work in a crowd environment. The issue of path planning thus extends far beyond a collision-free and shortest path if a care-receiver requires high-quality user experiences. A robot needs to obey 
social conventions and avert collision with human, in particular in walking. Path planning in dynamic environment aims at human-robot mutual understanding, i.e., social-aware path planning, with highlights such as comfort, naturalness and sociability.

A social-aware path planning framework typically contains the following components [40, 43, 44, 45]. First, a global planner provides to an robot the optimal path linking to the destination. Similar to traditional path planning like PFM, a global planner demands that a static map of the environment is at least partially prior known. Second, a local planner takes in charge collision avoidance with regards to moving obstacle. A typical method adopted by a local planner is the dynamic window approach (DWA). DWA prunes non-reachable velocity values and thus shrinks the searching space. Afterwards, DWA minimizes an objective function by choosing possible velocity values from the shrunk searching space. Third, prediction model will forecast human movements and further raise efficiency of path planning in a crowded dynamic environment. A simple way to predict human movement is leveraging the linear model where human motion trajectories mostly constitute of straight lines. An efficient social-aware path planning framework is required to properly unify all these components.

In terms of the three-component social-aware path planning framework, there still exist open questions. First, response should be as fast as possible. 60 For example, the local planner should swiftly adjust the routine of a fast-moving robot when an obstacle abruptly blocks the way. Second, new patterns of human motion may keep arising in the dynamic environment. Therefore, the local planner should be capable of updating collision avoidance models accordingly. This capability means a lifelong ability that can update a learning model using data collected. Third, it is a challenge to move in a crowded dynamic environment. In addition, it is even more complicated to plan the optimal paths for a swarm of robots, a trend in robotics with promising performance. The primary topic, in the case of swarm robotics, is fusing the latest refreshed collision avoidance models of all robots into one. Subsequently, all robots abide the fused model and achieve their globally optimal paths. 


\subsection{Psychotherapy}

Although psychological issues like depression are increasingly prevalent, many people still face high barrier to access mental healthcare. Mobile phone based socially assistant robot provides a promising solution to depress the accessibility nologies will speedup the progress toward practical robots.

Open research issues include intelligent communications, ground-breaking biosensors, cutting-edge AI and state-of-the-art deep learning algorithms. How- 


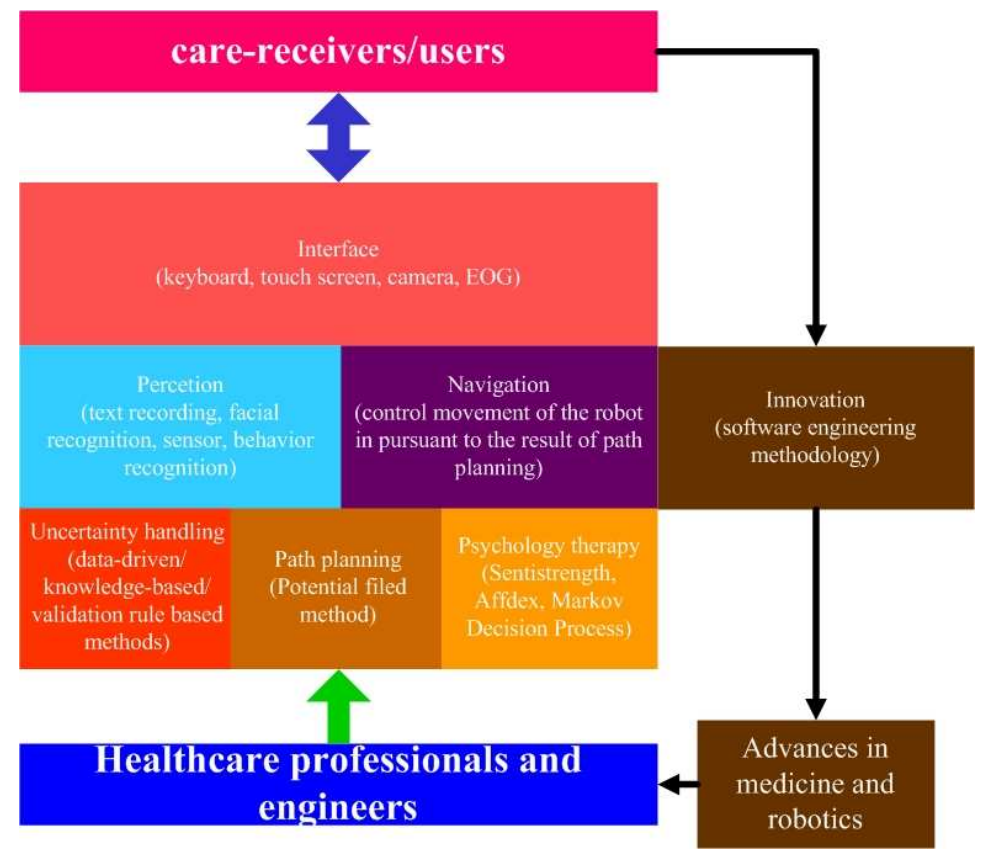

Figure 5: State-of-the-art supporting technologies of mobile healthcare robots

ever, each of the open issues still lacks advanced development, requiring further research and implementations. To this end, both academic and industrial research and development activities are highly recommended to overcome the limitations of the existing systems.

\section{Acknowledgments}

405 This work was supported in part by the National Natural Science Foundation of China under Grant 61672454; by the Fundamental Research Funds for the Central Universities of China under Grant 2722019PY052 and by the open project from the State Key Laboratory for Novel Software Technology, Nanjing University , under Grant No. KFKT2019B17.

[1] S. Jeong, and C. Breazeal. Toward Robotic Companions that Enhance Psychological Wellbeing with Smartphone Technology. Companion of the 2017 
ACM/IEEE International Conference on Human-Robot Interaction ACM. ACM, 2017, pp.345-346.

[2] P. Yang, D. Stankevicius, V. Marozas, Z. Deng, E. Liu. Lifelogging data validation model for internet of things enabled personalized healthcare. IEEE Transactions on Systems, Man, and Cybernetics Systems, preprint.

[3] V. Tao, K. Moy, V. Amirfar. A little robot with big promise may be future of personalized health care. Pharmacy Today, vol. 22. no.9, pp.38-38.

[4] Liu, Weiqing and Cao, Jiannong and Yang, Lei and Xu, Lin and Qiu, Xuanjia and Li, Jing. AppBooster: Boosting the Performance of Interactive Mobile Applications with Computation Offloading and Parameter Tuning. IEEE Transactions on Parallel and Distributed Systems, vol. 8. no.6, pp. 1593$1606,2017$.

[5] Sahni, Yuvraj and Cao, Jiannong and Zhang, Shigeng and Yang, Lei. Edge Mesh: A new paradigm to enable distributed intelligence in Internet of Things. IEEE Access, vol. 5, pp. 16441-16458, 2017.

[6] S Wan, Y Zhao, T Wang, Z Gu, QH Abbasi, KKR Choo, Multi-dimensional data indexing and range query processing via Voronoi diagram for internet of things, Future Generation Computer Systems 91, 382-391, 2019.

[7] Z Gao, DY Wang, SH Wan, H Zhang, YL Wang. Cognitive-inspired classstatistic matching with triple-constrain for camera free 3D object retrieval. Future Generation Computer Systems 94, 641-653, 2019.

[8] Dohr, A., Modreopsrian, R., Drobics, M., Hayn, D. and Schreier, G. The Internet of Things for Ambient Assisted Living. Seventh International Conference on Information Technology: New Generations, vol. 6,pp. 804-809, 2010.

[9] Miorandi, D., Sicari, S., Pellegrini, F. D., and Chlamtac, I. Internet of things: vision, applications and research challenges. Ad Hoc Networks, 10(7), pp. 1497-1516, 2012. 
[10] Chen, S., Xu, H., Liu, D., and Hu, B. A vision of iot: applications, challenges, and opportunities with china perspective. Internet of Things Journal IEEE, 1(4), pp. 349-359, 2014.

[11] Shi, W., Cao, J., Zhang, Q., Li, Y., and Xu, L. Edge computing: vision and challenges. Internet of Things Journal IEEE, 3(5), pp. 637-646, 2016.

[12] M. Carmen Domingo. An overview of the internet of things for people with disabilities. J. Netw. Comput. Appl., 35(2), pp. 584596, 2012.

[13] Hairong Yan, Li Da Xu, Zhuming Bi, Zhibo Pang, Jie Zhang, Yong Chen. An emerging technology a wearable wireless sensor networks with applications in human health condition monitoring. J. Manag. Anal., 2(2), pp. 121137, 2015.

[14] Pan, J., and Mcelhannon, J. Future edge cloud and edge computing for internet of things applications. IEEE Internet of Things Journal, Early Access, 2017.

[15] Z Gao, HZ Xuan, H Zhang, S Wan, KKR Choo. Adaptive fusion and category-level dictionary learning model for multi-view human action recognition. IEEE Internet of Things Journal, 2019.

[16] L Wang, H Zhen, X Fang, S Wan, W Ding, Y Guo. A unified two-parallelbranch deep neural network for joint gland contour and segmentation learning. Future Generation Computer Systems 100, 316-324, 2019. tion system. IEEE Trans. Ind. Inf. 10(2), pp. 15681577, 2014.

[18] Xu X., Li Y., Huang T., Xue Y., Peng K., Qi L., \& Dou W. An energyaware computation offloading method for smart edge computing in wireless metropolitan area networks. Journal of Network and Computer Applications, $133,75-85,2019$. 
[19] Xu X., Xue Y., Qi L., Yuan Y., Zhang X., Umer T., \& Wan S. An edge computing-enabled computation offloading method with privacy preservation for internet of connected vehicles. Future Generation Computer Systems, 96, 89-100, 2019.

[20] Lopez, P. G., Montresor, A., Epema, D., Datta, A., Higashino, T., and Iamnitchi, A., et al. Edge-centric computing: vision and challenges. Acm Sigcomm Computer Communication Review, 45(5), pp. 37-42, 2015.

[21] Tang, Ling and He, Shibo. Multi-User Computation Offloading in Mobile Edge Computing: A Behavioral Perspective. IEEE Network, 32(1), pp. 4853, 2018.

[22] Zhang, D., Chen, Z., Ren, J., Zhang, N., Awad, M. K., Zhou, H. and Shen, X. S. Energy-harvesting-aided spectrum sensing and data transmission in heterogeneous cognitive radio sensor network. IEEE Transactions on Vehicular Technology, 66(1), pp. 831-843, 2017.

[23] Yang, Lei and Liu, Bo and Cao, Jiannong and Sahni, Yuvraj and Wang, Zhenyu. Joint Computation Partitioning and Resource Allocation for Latency Sensitive Applications in Mobile Edge Clouds. Cloud Computing (CLOUD), 2017 IEEE 10th International Conference on, pp. 246-253, 2017.

[24] Lyu, Xinchen and Tian, Hui and Jiang, Li and Vinel, Alexey and Maharjan, Sabita and Gjessing, Stein and Zhang, Yan. Selective offloading in mobile edge computing for the green internet of things. IEEE Network, 32(1), pp. 54-60, 2018.

[25] W.Sanjaya, D. Anggraeni, R. Multajam, M. Subkhi, I, Muttaqien. Design and Experiment of Electrooculogram (EOG) System and Its Application to Control Mobile Robot. Materials Science and Engineering, vol.180, article no.012072, 2017.

[26] X. Wen, Y. Song, W. Li, G. Chen. Rotation Vector Sensor-Based Remote 
Control of a Mobile Robot via Google Glass. Robot Intelligence Technology and Applications, vol.447, pp.581-588, 2017.

[27] M. Uddin, M. Hassan, A. Almogren, A. Alamri, M. Alrubaian. Facial Expression Recognition Utilizing Local Direction-based Robust Features and Deep Belief Networ. IEEE Access, vol.5, pp.4525-4536, 2017.

[28] R. Liu, T. Chen, and L. Huang. Research on human activity recognition based on active learning. International Conference on Machine Learning and Cybernetics, IEEE, 2010, pp.285-290.

[29] Zhang, D, Shen, R, Ren, J. and Zhang, Y. Delay-optimal Proactive Service Framework for Block-Stream as a Service. IEEE Wireless Communications Letters, Early Access, 2018.

[30] LeCun Yann, Leon Bottou, Yoshua Bengio and Patrick Haffner. Gradientbased learning applied to document recognition. Proceedings of the IEEE. 86 (11): 22782324, 1998.

[31] Goodfellow, I. J., Pouget-Abadie, J., Mirza, M., Xu, B., Warde-Farley, D., and Ozair, S., et al. Generative adversarial networks. Advances in Neural Information Processing Systems, 3, pp. 2672-2680, 2014.

[32] T. Naegeli, J. Alonso-Mora, A. Domahidi, D. Rus, O. Hilliges. Real-Time Motion Planning for Aerial Videography With Real-Time With Dynamic Obstacle Avoidance and Viewpoint Optimization. IEEE Robotics and Automation Letters, vol.2, no.3, pp.1696-1703, 2017.

[33] M. Castro, D. Mulero, M. Ferre. A Real-Time Reconfigurable Collision Avoidance System for Robot Manipulation. the 3rd International Conference on Mechatronics and Robotics Engineering. ACM, 2017, pp.6-10.

[34] C. Morais, T. Nascimento, A. Brito, G. Basso. A 3D Anti-collision System based on Artificial Potential Field Method for a Mobile Robot. International Conference on Agents and Artificial Intelligence. Scitepress, 2017, pp.308313. 
[35] Zhang, D., Chen, Z., Awad, M., Zhang, N., Zhou, H. and Shen, X. S. Utility-optimal resource management and allocation algorithm for energy harvesting cognitive radio sensor networks. IEEE Journal on Selected Areas in Communications. 34(12), 2017, pp.3552-3565.

[36] M. Weninger, M. Weninger, M. Weninger, C. Gisinger, S. Frennert. Results of a real world trial with a mobile social service robot for older adults. ACM/IEEE International Conference on Human-Robot Interaction. IEEE, 2016, pp.497-498.

[37] D. Iannuzzi, A. Grant, H. Corriveau, P. Boissy, F. Michaud, Specification of an Integrated Information Architecture for a Mobile Teleoperated Robot for Home Telecare, Informatics for Health and Social Care, vol.41, no.4, pp.350-361, 2016.

[38] H. Sfar, A. Bouzeghoub, Nathan Ramoly,Jerme Boudy. Agacy monitoring: a hybrid model for activity recognition and uncertainty handling. Lecture Notes in Computer Science, 2017.

[39] M. Noor, Z. Salcic, I. Wang. Enhancing ontological reasoning with uncertainty handling for activity recognition. Knowledge-Based Systems, vol.114, pp.47-60, 2016.

[40] S. Chik, C. Yeong, E. Su. A review of social-aware navigation frameworks for service robot in dynamic human environments. Journal of Telecommunication, Electronic and Computer Engineering, vol.11 no.8,pp.41-50,2016.

[41] Salahuddin, M. A., Al-Fuqaha, A., and Guizani, M. Software-defined networking for rsu clouds in support of the internet of vehicles. IEEE Internet of Things Journal, vol.2 no.2,pp. 133-144,2015.

[42] Sood, K., Yu, S., and Xiang, Y. Software-defined wireless networking opportunities and challenges for internet-of-things: a review. IEEE Internet of Things Journal, vol.3 no.4,pp. 453-463, 2016. 
[43] Yuyu Yin, Lu Chen, Yueshen Xu, Jian Wan, He Zhang, Zhida Mai. QoS Prediction for Service Recommendation with Deep Feature Learning in Edge Computing Environment. Mobile Networks and Applications. 2019. https://doi.org/10.1007/s11036-019-01241-7.

[44] Honghao Gao, Wanqiu Huang, Xiaoxian Yang, Yucong Duan, Yuyu Yin. Towards Service Selection for Workflow Reconfiguration: An Interface-Based Computing. Future Generation Computer Systems(FGCS). 2018,87:298-311.

[45] Honghao Gao, Shunyi Mao, Wanqiu Huang, Xiaoxian Yang. Applying Probabilistic Model Checking to Financial Production Risk Evaluation and Control: A Case Study of Alibaba's Yu'e Bao. IEEE Transactions on Computational Social Systems(TCSS).2018, 5(3):785-795.

[46] Yuyu Yin, Fangzheng Yu, Yueshen Xu, Lifeng Yu, Jinglong Mu, Network Location-Aware Service Recommendation with Random Walk in CyberPhysical Systems. Sensors 17(9): 2059 (2017)

[47] Chen, Y., Deng, S., Ma, H. et al. Deploying Data-intensive Applications with Multiple Services Components on Edge. Mobile Networks and Applications (2019). https://doi.org/10.1007/s11036-019-01245-3.

[48] W Li, X Liu, J Liu, P Chen, S Wan, X Cui. On Improving the accuracy with Auto-Encoder on Conjunctivitis, Applied Soft Computing 81, 105489, 2019.

[49] S Ding, S Qu, Y Xi, AK Sangaiah, S Wan. Image caption generation with high-level image features, Pattern Recognition Letters 123, 89-95, 2019.

[50] Y Zhao, H Li, S Wan, A Sekuboyina, X Hu, G Tetteh, M Piraud, B Menze. Knowledge-aided convolutional neural network for small organ segmentation. IEEE journal of biomedical and health informatics, 2019.

[51] Y Xia, S Qu, S Wan. Scene guided colorization using neural networks. Neural Computing and Applications, 1-14, 2019. 
[52] Xin Yi, Ekta Walia, Paul Babyn. Generative Adversarial Network in Medical Imaging: A Review. Medical Image Analysis, 2019,58, 101552.

[53] S. Ding, S. Qu, Y. Xi, S. Wan. Stimulus-driven and concept-driven analysis for image caption generation. Neurocomputing, 2019.

[54] R. Zhang, P. Xie, C. Wang, G Liu, S. Wan. Classifying transportation mode and speed from trajectory data via deep multi-scale learning. Computer Networks, 2019, 162, 106861.

[55] Zackory M. Erickson, Sonia Chernova, Charles C. Kemp. Semi-Supervised Haptic Material Recognition for Robots using Generative Adversarial Networks. CoRL 2017: 157-166. 